Research Paper

\title{
An Innovative Strategy for the Fabrication of Functional Cell Sheets Using an Electroactive Conducting Polymer
}

\author{
HyungJae Lee and Youngnam Cho ${ }^{凶}$ \\ New Experimental Therapeutic Branch, National Cancer Center, 111 Jungbalsan-ro, Ilsandong-gu, Goyang, Gyeonggi-do 410-769, South Korea \\ $\triangle$ Corresponding author: E-mail: yncho@ncc.re.kr
}

() 2015 Ivyspring International Publisher. Reproduction is permitted for personal, noncommercial use, provided that the article is in whole, unmodified, and properly cited. See http://ivyspring.com/terms for terms and conditions.

Received: 2015.04.13; Accepted: 2015.05.09; Published: 2015.06.10

\begin{abstract}
Here, we report the development of an electric field-assisted methodology for constructing 3D $\mathrm{C} 2 \mathrm{Cl} 2$ cell sheets with the potential for cell surface modification. In this method, a conducting polymer, polypyrrole (Ppy), is electrodeposited via biotin doping, and then chemical conjugation of biotinylated bone morphogenetic protein 2 (BMP2) is achieved using a biotin-streptavidin cross-linker. Subsequently, $\mathrm{C} 2 \mathrm{Cl} 2$ cells are cultured on BMP2-immobilized Ppy surfaces to induce interactions between cell surface receptors and bound BMP2 ligands. Following these procedures, layers of BMP2-immobilized cells can be easily detached from the Ppy surface by applying an electrical potential. This novel method results in high affinity, ligand-bound cell sheets, which exhibit homogeneous coverage with membrane-bound proteins and signal activation that occurs via maximal receptor accessibility. Using this strategy to engineer the cell surface with desirable ligands results in structures that mimic in vivo tissues; thus, the method reported here has potential applications in regenerative medicine and tissue engineering.
\end{abstract}

Key words: cell sheet, conducting polymer, osteogenesis, bone morphogenetic protein 2, electrical stimulation

\section{Introduction}

In recent decades, significant efforts have been directed toward developing effective methods for the treatment of tissue and organ dysfunction or failure. The traditional clinical approach involves cell-based therapies, in which autologous cells are implanted or injected directly into target sites. ${ }^{[1-4]}$ However, difficulties associated with the anchorage and adaptation of dissociated cells to the target tissues have hindered the practical use of these methods. ${ }^{[5,6]}$ Tissue engineering, in which cells and growth factors are organized into 3D scaffolds, offers an alternative approach. Tissues and organs are composed of a complex 3D network comprising cells, extracellular matrix (ECM), and signaling molecules. The cell-cell and cell-ECM interactions in these networks are important for regulating biochemical and cellular responses. Tissue engineering aims to mimic these natural biological functions without disrupting them.
However, achieving this aim requires biocompatible scaffolds that act as structural templates and promote cellular adhesion, cellular proliferation, and eventually tissue formation. In general, synthetic and natural biomaterials are employed as ECM-like scaffolds, which serve as a matrix for uniform cell seeding and adhesion, and for controlling the release of various growth factors. ${ }^{[7-9]}$ Recently, however, cell sheet engineering has been proposed as a "scaffold-free" tissue engineering approach, which could be particularly advantageous when a temperature-responsive polymer is used.[10-13] Compared to the injection of isolated cells, this scaffold-free method improves cell adhesion and proliferation, and thus improves integration with host tissues; concurrently, the original function, architecture, and integrity of the ECM are maintained. Scaffold-free cell sheet technology has been applied for regeneration of damaged tissues and 
organs in various animal models as well as in clinical trials involving the esophagus, corneas, and myocardia.[14-17] Despite their advantages, the use of cell sheets presents certain challenges. For example, in order to analyze the in vitro/in vivo activity of cell sheets, it is necessary to induce biochemical and cellular responses by exogenous administration of growth factors. However, the cells may receive insufficient levels of growth factor because of rapid diffusion from the target site following soluble delivery, and this can interfere with the interactions and communication of receptors and ligands. Recently, we demonstrated the performance of a conducting polymer, polypyrrole (Ppy), as a highly efficient cell capture/release platform. ${ }^{[18-20]}$ According to our previous studies, Ppy is capable of encapsulating biotin in a polymeric backbone by oxidation and subsequently releasing entrapped molecules via reduction. In the current study, we applied the intrinsic electroactive nature of Ppy to develop a novel scaffold-free cell sheet technology. By engineering the cell surface with desirable ligands, we were able to produce structures that mimicked in vivo tissues. Therefore, the method described here could potentially be applied in regenerative medicine and tissue engineering.

\section{RESULTS AND DISCUSSION}

\section{C2C12 cell sheets specifically conjugated with bone morphogenetic protein 2 (BMP2)}

A schematic diagram of the fabrication process for the 3D cell sheets is shown in Figure 1. Initially, Ppy was electrochemically polymerized on an ITO surface by using biotin as a co-dopant in the Ppy film. Biotin can be employed as a bridge in conjugation with target biomolecules. With this approach, it was possible to fabricate electric-field-assisted cell sheets, in which the mouse skeletal muscle-derived $\mathrm{C} 2 \mathrm{C} 12$ cell line was used as a working model. Importantly, individual cells within the 3D constructs could be efficiently tethered with growth factors, specifically bone morphogenetic protein 2 (BMP2), via cell surface receptors. BMP2 plays an important role in inducing osteoblastic differentiation of the $\mathrm{C} 2 \mathrm{C} 12$ myoblasts by blocking the myogenic differentiation pathway. ${ }^{[21,22]}$ The introduction of BMP2 in the vicinity of the cell surface increases the recognition of, as well as communication with, cell membrane receptors, which facilitates the stable formation of complexes between growth factors and receptors with sustained receptor activation. This strategy allows for the manipulation of individual target cells with desired functional entities and, subsequently, the modulation of cellular activity. Indeed, the guided assembly of biotin as a dopant is expected to achieve the homogeneous and spatial arrangement of target moieties. The advantages of the Ppy-based scaffold-free cell sheets produced in this study are as follows: (i) the spontaneous and reversible red-ox reaction of the biotin-doped Ppy surface enables controlled incorporation and release of biomolecules; (ii) successful binding of BMP2 with receptors on the extracellular side of the cellular membrane significantly enhances the osteogenic differentiation of $\mathrm{C} 2 \mathrm{C} 12$ cells, and does so by efficient and site-specific delivery of functional proteins; (iii) the scaffold-free technology promotes improved integration with the surrounding tissues, mimicry of tissue function, and high cell density that together lead to more complete regeneration of tissue. The simplicity of its fabrication should allow our scaffold-free cell sheet to be integrated with in vitro tissue/organ models and be used in vivo cell-based therapy.

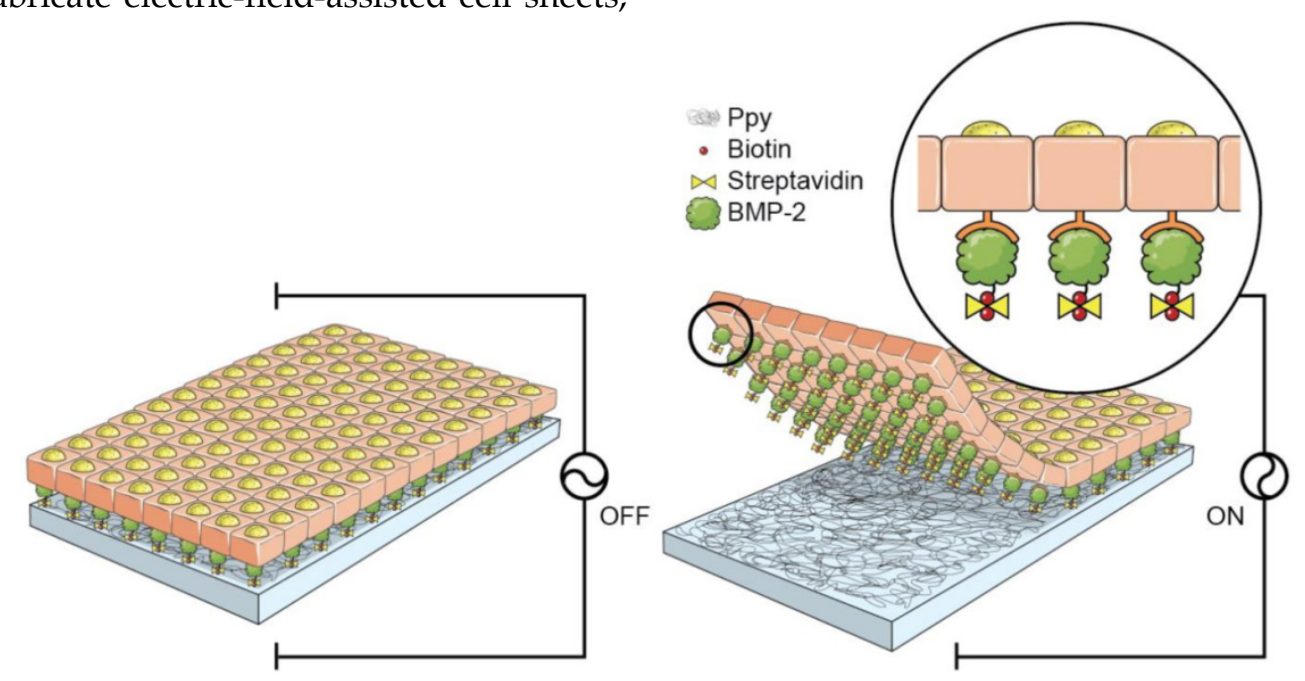

Figure 1. Schematic diagram of the fabrication process for 3D cell sheets specifically conjugated with bone morphogenetic protein 2 (BMP2). C2C12 cells were incubated on BMP2-immobilized, biotin-doped polypyrrole (Ppy) surfaces. Cell sheets were produced with individual cells efficiently tethered to growth factors (specifically BMP2) via cell surface receptors, and these could be non-destructively released from Ppy by applying an electric field (-0.8 $\vee$ for $30 \mathrm{~s}$ ). Recovered cell sheets could be made to overlap as a 3D-multilayer. 
A

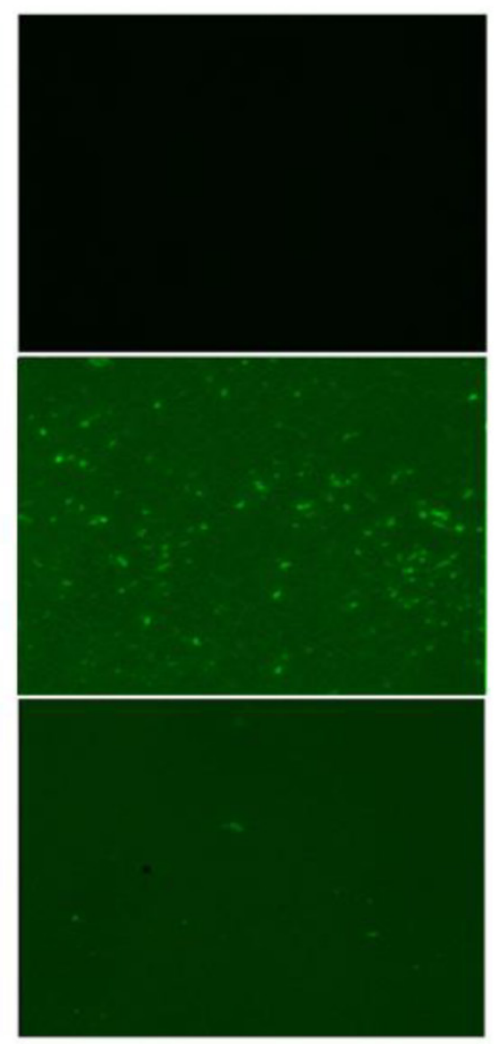

B

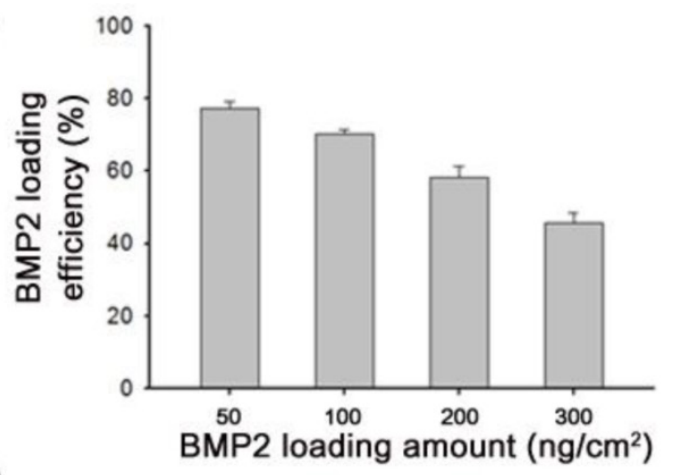

C
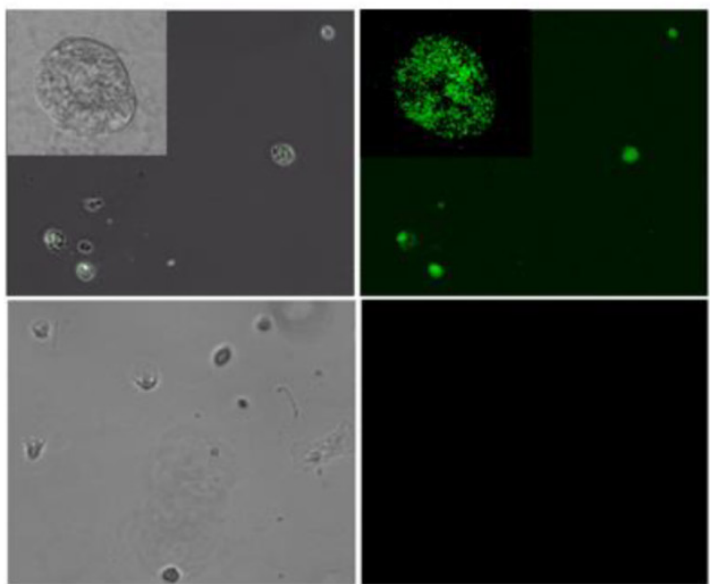

Figure 2. (A) Fluorescence microscopy images of polypyrrole (Ppy) only (top), and bone morphogenetic protein 2 (BMP2)-immobilized biotin-doped Ppy before (middle) and after (bottom) electrical stimulation. Electrical potential ( $-0.8 \vee$ for $30 \mathrm{~s})$ induced massive release of biotin and conjugated BMP2 from Ppy. Surface-immobilized BMP2 was visualized using fluorescein isothiocyanate (FITC)-conjugated anti-BMP2 antibody. (B) Quantification (by ELISA) of BMP2 immobilized on biotin-doped Ppy surfaces by using various protein concentrations. (C) Fluorescence images of BMP2-conjugated C2C12 cells (upper panels) and normal C2C12 cells (lower panels). After electrical stimulation, the detached BMP2-conjugated cells were incubated in a solution containing anti-BMP2 labeled FITC.

\section{Loading efficiency of BMP2 on Ppy platform}

Here, we explored the feasibility of this approach by investigating the efficacy of immobilization of biotinylated BMP2 to the functionalized Ppy surface. In general, $\mathrm{C} 2 \mathrm{C} 12$ cells are cultured in medium containing BMP2. Under these circumstances, soluble delivery of proteins often restricts their availability to cell surface receptors. We attempted to overcome this problem by constructing cell sheets with BMP2 ligands tagged to individual cells. Initially, we analyzed the binding efficacy of BMP2 to biotin-doped Ppy by using fluorescence microscopy; fluorescence images were used to examine the distribution of surface-immobilized proteins, with fluorescein isothiocyanate (FITC)-conjugated anti-BMP2 employed as a detection probe (Figure 2A). The green fluorescent regions visualized on Ppy surfaces indicated the existence of BMP2 because of the preferential association of the biotin-streptavidin linkage. However, in response to an applied electric field, the fluorescent signals disappeared from the electrically stimulated Ppy surface, which can primarily be explained by the massive release of biotin and conjugated BMP2 from
Ppy. We also examined the BMP2 loading efficacy by using various concentrations. As shown in Figure 2B, when $50-300 \mathrm{ng} / \mathrm{cm}^{2}$ BMP2 was applied onto the biotin-doped Ppy surfaces, the amount of immobilized BMP2 (quantified by ELISA) was $39-147 \mathrm{ng} / \mathrm{cm}^{2}$, suggesting that the levels of surface-immobilized BMP2 would be sufficient to enhance its availability to cell surface receptors. In addition, we demonstrated the presence of BMP2 on the surface of $\mathrm{C} 2 \mathrm{C} 12$ cells (Figure 2C). By applying an electric field, we released cells specifically bound with growth factors to membrane receptors. BMP2-conjugated $\mathrm{C} 2 \mathrm{C} 12$ cells were then incubated in a solution containing anti-BMP2 labeled with FITC. Finally, BMP2 was observed along the cell membranes of BMP2-conjugated $\mathrm{C} 2 \mathrm{C} 12$ cells but not in normal C2C12 cells (Figure 2C). In the present study, we proposed that individual cells within a cell sheet could be labeled with BMP2 to stimulate osteogenesis in $\mathrm{C} 2 \mathrm{C} 12$ myogenic cells. Because the biotin within the Ppy surface allows greater flexibility for the incorporation of new biological moieties, this could be a versatile and molecularly well-defined methodology for cell surface engineering and may be compatible with a variety of cellular applications. 


\section{Fabrication of BMP2-immobilized $\mathrm{C} 2 \mathrm{C} 12$ cell sheets using biotin-doped Ppy platform}

As shown in Figure 3, we also demonstrated the construction of BMP2-conjugated $\mathrm{C} 2 \mathrm{C} 12$ cell sheets. First, C2C12 cells $\left(5 \times 10^{4}\right)$ were seeded on a BMP2-immobilized biotin-doped Ppy surface for $5 \mathrm{~d}$ to facilitate a tight interface between cells. The cells assembled as a sheet could be non-destructively detached from the Ppy surface by applying electric fields (Figure 3A). Indeed, a weak electric potential delicately modulated the unique cell-surface interface by causing the spontaneous release of biotin moieties and attached cells from the surface. Recovered cell sheets could be easily fabricated as 3D cellular multilayers. We also investigated the effect of electric potentials on manipulating cell sheet technology by applying electrical fields ranging from $-0.8 \mathrm{~V}$ to $+0.4 \mathrm{~V}$ to the Ppy surfaces for $30 \mathrm{~s}$ (Figure 3B). Consistent with our previous studies, ${ }^{[18,19]}$ the monolayered cell sheet was readily detached from the surface by gentle agitation with a PBS-filled pipette following negative electrical stimulation. However, electrically positive stimulation of Ppy surfaces produced no reaction, with results that were similar to those of the unstimulated control. We further evaluated the electrochemical behavior of Ppy surfaces by using cyclic voltammetry $(\mathrm{CV})$, as shown in Figure 3C. Redox peak currents were enhanced on the biotin-doped Ppy surface. However, $\mathrm{C} 2 \mathrm{C} 12$ cell sheets grown on BMP2-immobilized, biotin-doped Ppy surfaces exhibited obviously decreased electron-transfer capabilities, specifically of electroactive ferricyanide species throughout the Ppy surfaces, because of tight cell-surface junctions. Electrical stimulation at $-0.8 \mathrm{~V}$ was sufficient to detach the cell sheet from the Ppy surface, which could ultimately restore the current intensity to its original state by allowing the free transfer of electrolytes. We noted, however, that a positive potential did not affect peak intensity, which indicates that $\mathrm{C} 2 \mathrm{C} 12$ cell sheets remained firmly attached to the Ppy surface regardless of the applied electrical stimulation. Electrical stimulation induces the conformational change of Ppy backbones via oxidation/reduction reactions; specifically, the Ppy polymer swells significantly with a positive potential, generating free volume and enabling entrapment of various moieties inside the polymeric backbone. ${ }^{[23]}$ In contrast, at negative potentials, Ppy undergoes structural shrinkage and squeezes out molecules incorporated within the polymer. Indeed, our results demonstrated preferential detachment of cell sheets only when negative electrical potentials were applied.

A

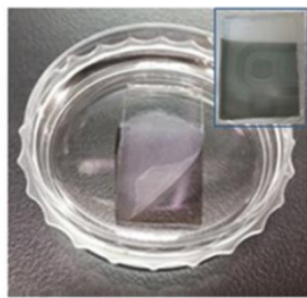

B

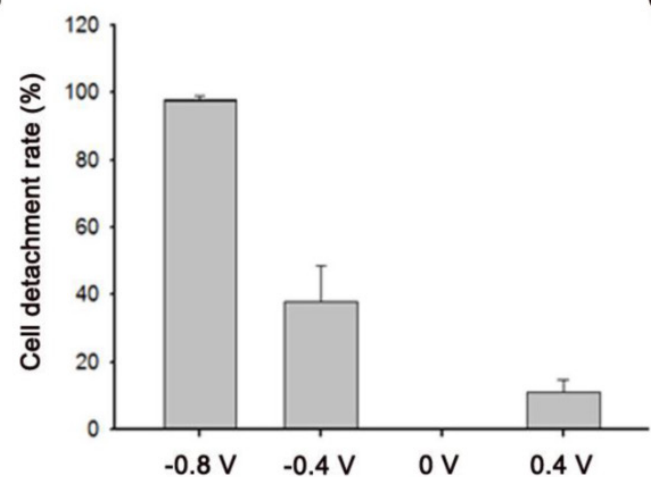

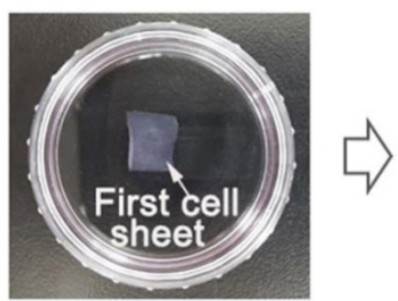

C
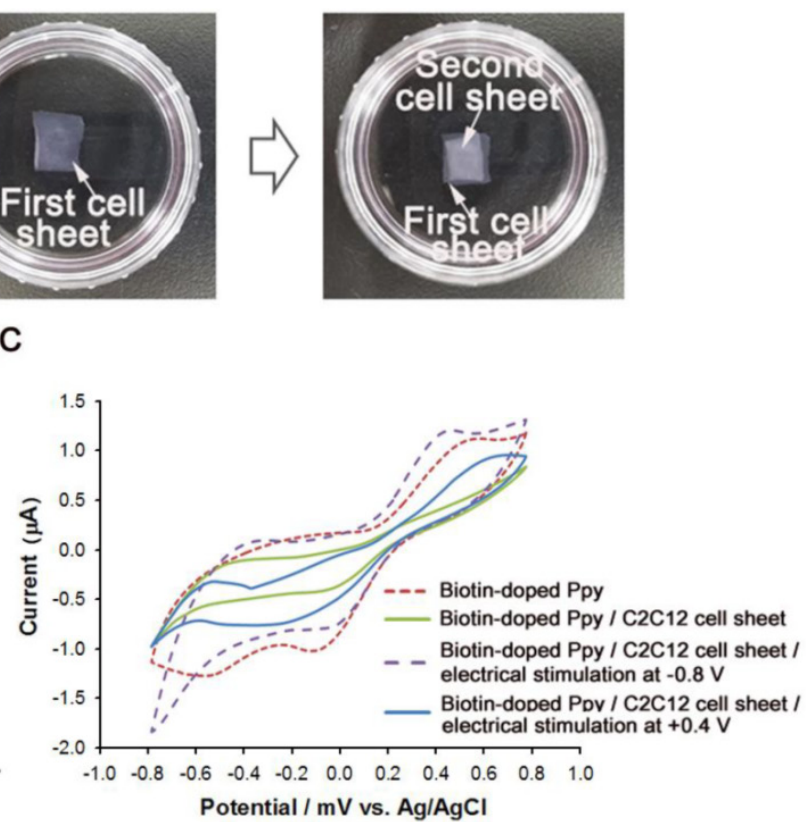

Figure 3. (A) Bright-field photography of $\mathrm{C} 2 \mathrm{Cl} 2$ cell sheet(s) detached from biotin-doped polypyrrole (Ppy) in response to electrical stimulation (-0.8 $\mathrm{V}$ for $30 \mathrm{~s}$ ). Inset image shows Ppy surface after the detachment of the cell sheet. 3D cell sheets were fabricated by repeatedly layering detached cell sheets in a 35-mm cell culture dish. (B) Efficiency of cell detachment from bone morphogenetic protein 2 (BMP2)-immobilized biotin-doped Ppy in response to electric fields (+0.4 $\mathrm{V}$ to $-0.8 \mathrm{~V}$ for $30 \mathrm{~s}$ ). (C) Effect of electrical stimulation on the cyclic voltammogram curve, with a 5-mM ferricyanide probe solution used as an indicator. 
A

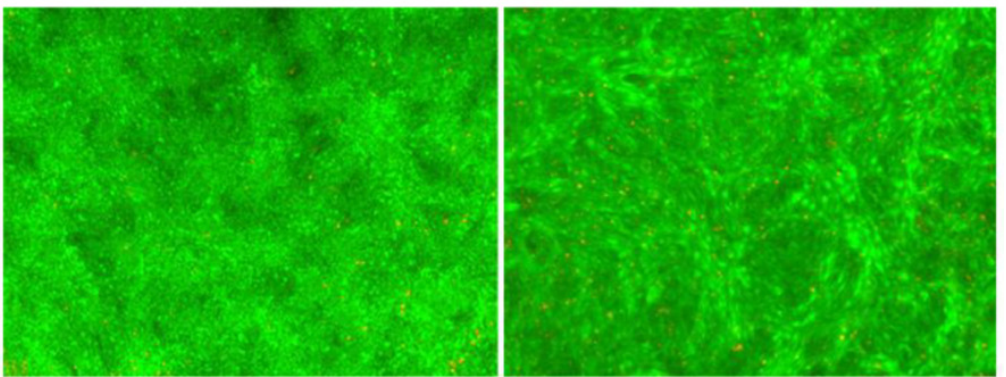

B

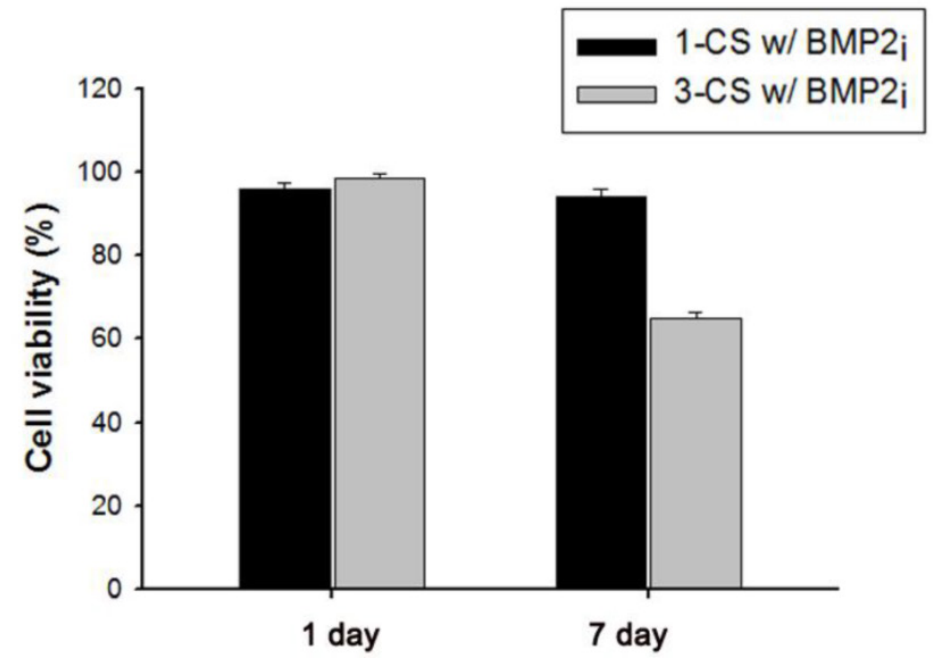

Figure 4. (A) Fluorescence-based live/dead viability assay of one-layered, BMP2-immobilized C2C12 cell sheets (1-CS w/BMP2 $2_{i}$ after $1-d$ (left) or 7-d (right) cultivation. (B) Relative cell viability of one-layered (1-CS w/BMP2 $2_{i}$ ) and three-layered (3-CS w/BMP2 $2_{i}$ BMP2-immobilized cell sheet(s) after $1-d$ or 7-d cultivation.

\section{Characterization of BMP2-immobilized C2C12 cell sheets}

We also investigated the viability of monolayered or multilayered cell sheet(s) after $1 \mathrm{~d}$ and $7 \mathrm{~d}$ of incubation, and we found that $\mathrm{C} 2 \mathrm{C} 12$ cells remained healthy even after $7 \mathrm{~d}$ (Figure 4A-B). In addition, the morphology of a monolayer of cells was investigated using phase contrast and fluorescence microscopy (Figure 5A-F). In BMP2-labeled C2C12 cell sheets, cells were round in shape and exhibited an osteogenic phenotype after only $4 \mathrm{~d}$, which was a pattern comparable to that of cells from culture media where an equivalent amount of BMP2 had been added. In contrast, cells cultured in a conventional culture dish without BMP2 exhibited a normal spindle-shaped morphology. We also used confocal laser scanning microscopy to observe a bilayer composed of $\mathrm{C} 2 \mathrm{C} 12$ cells (Figure 5G). After sequential electrical stimulation, the recovered second cell layer overlapped with the first cell layer, thereby mimicking 3D tissue formation. The fabricated cell sheets had an approximate thickness of $35 \mu \mathrm{m}$. We explored the effect of BMP2-immobilized C2C12 cell sheets on osteoblastic expression. After electrical stimulation, BMP2-immobilized cell sheets (CS $\mathrm{w} / \mathrm{BMP}_{\mathrm{i}}$ ) were transferred to a culture dish to assess alkaline phosphatase (ALP) activity in the C2C12 cells (Figure 6A). ALP activity in single-layered BMP2-immobilized cell sheets (1-CS w/BMP2 $2_{\mathrm{i}}$ ) apparently improved 4-fold compared with that in $\mathrm{C} 2 \mathrm{C} 12$ cells cultured in BMP2-free media (CS w/o BMP2 2 ). Additionally, ALP activity was significantly higher in multilayered sheets than single layers, which indicated that 3D tissues created by stacking individual cell sheets could be more therapeutically effective when transplanted. In comparison with cell sheets cultured in BMP2-added media (CS w/BMP2 $2_{\mathrm{a}}$ ), the enhanced ALP activity in CS $\mathrm{w} / \mathrm{BMP}_{2}$ could be attributed to membrane-bound growth factors, which achieve receptor-ligand complex formation. Growth factor-tethered cell sheets showed efficient cellular activity that was significantly correlated with the defined amount of BMP2 present in individual cells, which would be unlikely with traditional soluble delivery methods. Indeed, direct binding of biomolecules to individual cells yields uniform distribution and long-term contact duration by restricting diffusion from the integration site, and thereby efficiently triggers differentiation. We also examined osteoblastic differentiation of $\mathrm{C} 2 \mathrm{C} 12$ cells within 3D cell constructs by using an Alizarin red staining assay (Figure 6B-C). 
Interestingly, $\mathrm{CS} \mathrm{w} / \mathrm{BMP}_{\mathrm{i}}$ showed more intense red staining than $\mathrm{CS} w / \mathrm{BMP}_{\mathrm{a}}$ in both normal cell media and osteogenic media. The results of Alizarin red staining suggested that BMP2-bound cell sheets favorably affect the induction of osteogenic differentiation and increase the accumulation of mineralized calcium phosphate. We found that $\mathrm{CS} \mathrm{w} / \mathrm{BMP}_{\mathrm{i}}$ induced a 4-fold increase in mineral deposition compared to CS $\mathrm{w} / \mathrm{BMP} 2_{\mathrm{a}}$, especially in osteogenic media.

\section{CONCLUSIONS}

In conclusion, we developed BMP2-immobilized C2C12 cell sheets without using an artificial scaffold. The electroactive, biotin-doped Ppy surfaces of these cell sheets were capable of conjugating BMP2 at biologically relevant levels via biotin-streptavidin interaction. After using ELISA to quantify immobilized BMP2 on Ppy surfaces, we incubated $\mathrm{C} 2 \mathrm{C} 12$ cells on Ppy to (i) strengthen the cell-cell junction and (ii) modify cell surface receptors with BMP2 ligands. Following electrical stimulation at a negative potential, BMP2-bound cell sheets were non-destructively detached and transferred to culture dishes, where their osteogenic differentiation capabilities were assessed. Our results indicate that this approach could be a valuable and flexible tool for attaching bioactive molecules to cell surfaces in applications that require cell culture, tissue engineering, or both. Because cellular function could be affected by the immobilized proteins, in future research we will apply this technique to induce multipotent differentiation of human mesenchymal stem cells and investigate the different phenotypes and functions.

\section{EXPERIMENTAL SECTION}

\section{Materials}

Pyrrole, sodium dodecylbenzene sulfonate (NaDBS), biotin, 1-ethyl-3-(3-dimethylaminopropyl) carbodiimide (EDC), and N-hydroxysuccinimide (NHS) were purchased from Sigma-Aldrich. Recombinant human BMP2 was obtained from Peprotech.

\section{Fabrication of BMP2-immobilized polypyrrole (Ppy) platform}

The BMP2-immobilized Ppy platform was prepared electrochemically using a potentiostat/galvanostat (BioLogic SP-50). ITO, platinum wire, and $\mathrm{Ag} / \mathrm{AgCl}$ reference were employed as the working, counter, and reference electrodes, respectively. In order to prepare a biotin-doped Ppy platform with a surface area of $1 \mathrm{~cm}^{2}$, pyrrole was polymerized in $0.1 \mathrm{M}$ pyrrole and $0.01 \mathrm{M} \mathrm{NaDBS}$ solution by adding $1 \mathrm{mM}$ of biotin and applying chronoamperometry (CA) at $0.8 \mathrm{~V}$ for $60 \mathrm{~s}$. Subsequently, the biotin-doped Ppy platforms were washed three times with ultrapure water. After air-drying, $10 \mathrm{ng} / \mathrm{mL}$ of streptavidin (SA) was conjugated with biotin on the Ppy platform for $30 \mathrm{~min}$ and then washed with ultrapure water. Various concentrations $(50,100,200$, and $300 \mathrm{ng}$ ) of BMP2 were then biotinylated using Sulfo-NHS-Biotin (Thermo Scientific) solution according to the manufacturer's protocol and added to the SA-terminated Ppy surface.

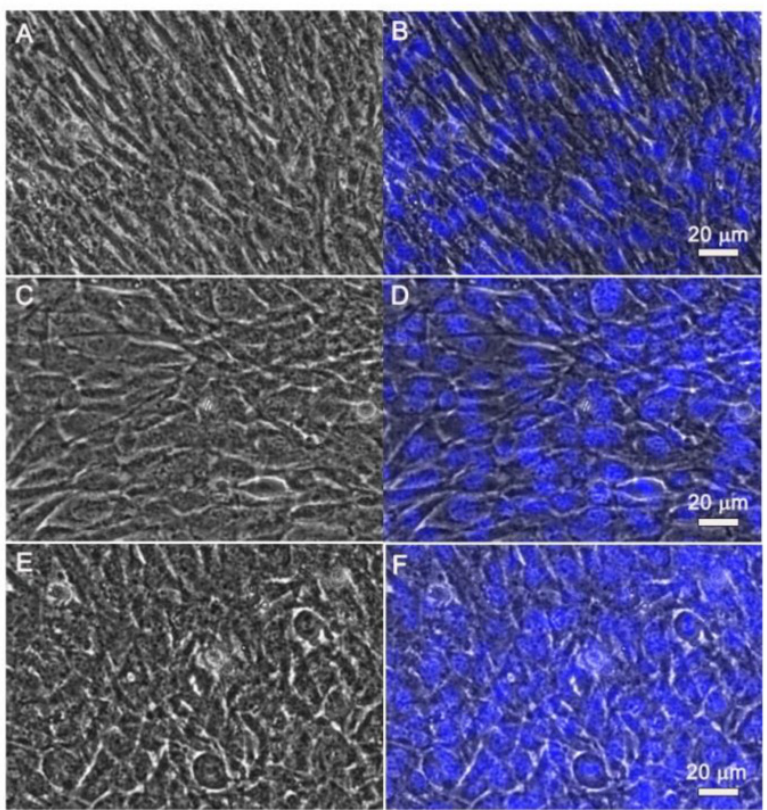

G
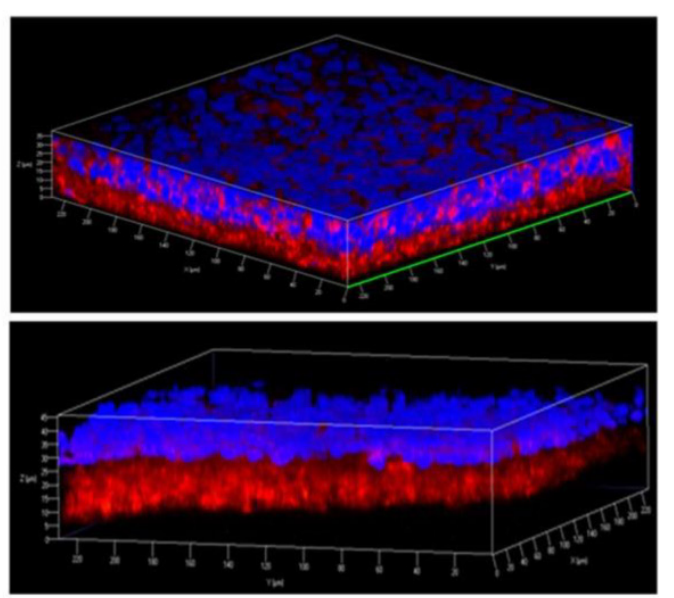

Figure 5. Phase contrast and fluorescence images of $(A-B) C 2 C 12$ cell sheet as a control, (C-D) $\mathrm{C} 2 \mathrm{Cl} 2$ cell sheet with $100 \mathrm{ng}$ of bone morphogenetic protein 2 (BMP2) in culture media, and (E-F) $\mathrm{C} 2 \mathrm{C} 12$ cell sheet immobilized with BMP2. After a 7-d incubation, cell sheets were detached with electrical stimulation. Fluorescence images were obtained by nuclei labeling with Hoechst dye. (G) Confocal laser scanning microscopy images of double-layered cell sheets. The first recovered cell sheet was stained with streptavidin Cy3 after cell biotinylation and the second recovered cell sheet was stained with Hoechst dye. 
A

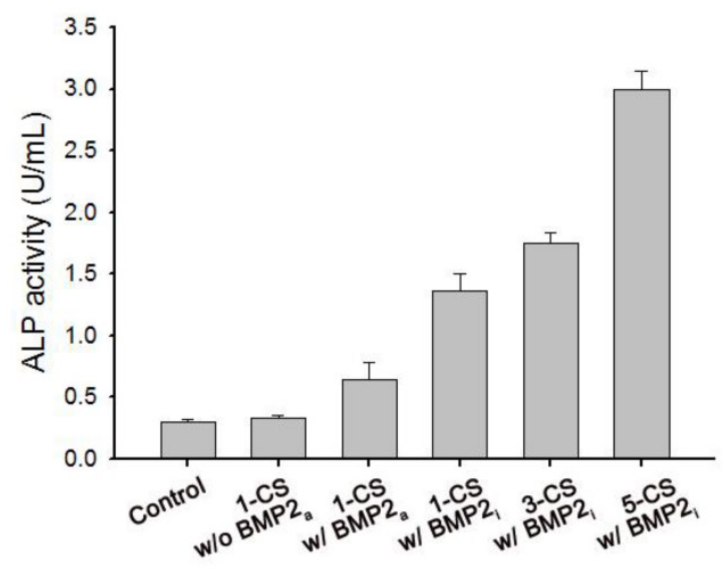

C

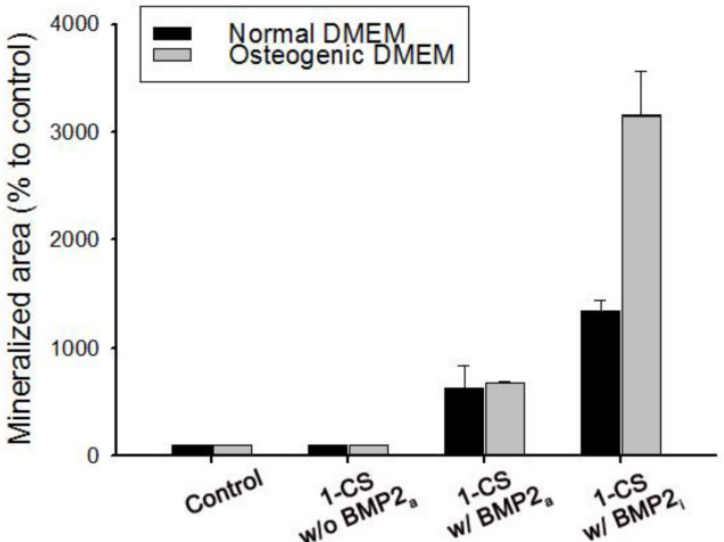

B

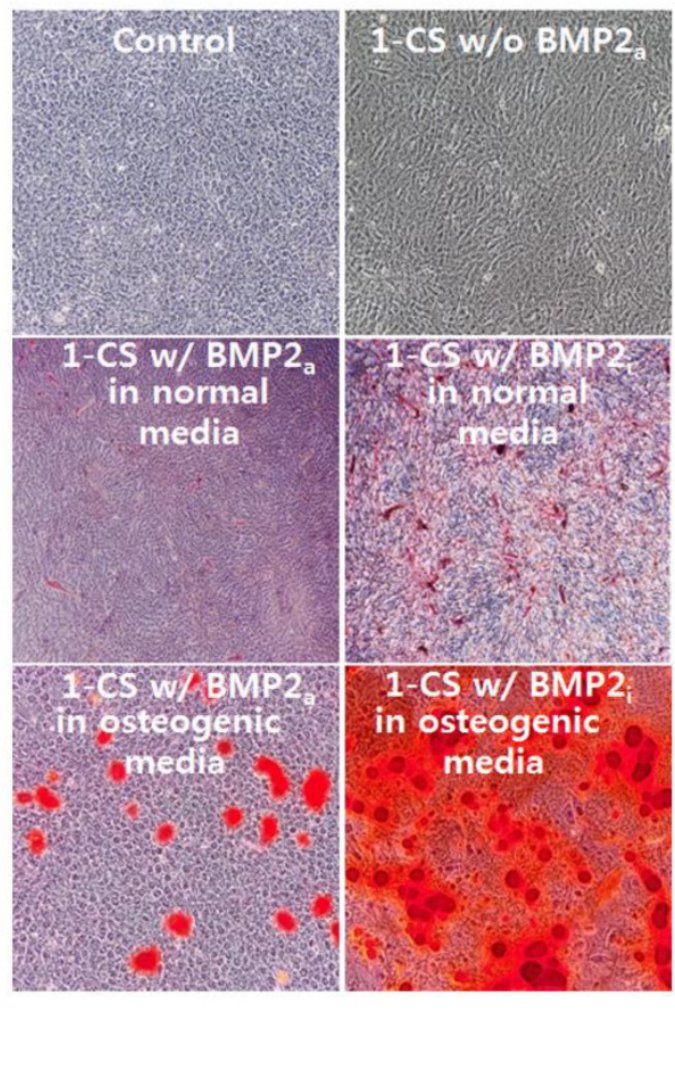

Figure 6. (A) Alkaline phosphatase (ALP) activity of (i) one-layered $\mathrm{C} 2 \mathrm{C} 12$ cell sheet as a control, (ii) one-layered $\mathrm{C} 2 \mathrm{C} 12$ cell sheet without addition of bone morphogenetic protein 2 (BMP2) in culture media (1-CS w/o BMP2 2 ), (iii) one-layered $C 2 C 12$ cell sheet with $100 \mathrm{ng}$ of $B M P 2$ in culture media (1-CS w/BMP2 $2_{a}$ ), and (iv) one-layered, three-layered, and five-layered $\mathrm{C} 2 \mathrm{Cl} 2$ cell sheet(s) immobilized with BMP2 (1-, 3-, and 5-CS w/BMP2 $2_{i}$. (B) Alizarin red staining of mono-layered cell sheets in normal DMEM or osteogenic DMEM for $7 \mathrm{~d}$. BMP2-immobilized cell sheets (CS w/BMP2 $2_{i}$ ) were stained more intensely red than BMP2-added cell sheets (CS w/BMP2 $2_{a}$ ) in both normal cell media and osteogenic media. (C) Quantification of mineralized area (red stained) by using the Image J software, where the stained areas indicate osteoblast mineralization.

\section{Loading efficiency of BMP2 on Ppy platform}

0, 100, 200 and 300 ng of BMP2-immobilized Ppy platforms $(50,100,200$, and $300 \mathrm{ng})$ were electrically stimulated via the application of CA at $-0.8 \mathrm{~V}$ for $30 \mathrm{~s}$ to induce the release of BMP2 from the surface. The amount of released BMP2 in supernatants was assayed and quantified using with Quantikine BMP-2 ELISA kit (R\&D Systems). For visualization of the BMP2 attached on the Ppy surface, $30 \mu \mathrm{L}$ of FITC-conjugated anti BMP-2 antibody $(5 \mu \mathrm{g} / \mathrm{mL}$ in PBS, Abcam) was added to the BMP2-loaded Ppy surfaces and incubated for $6 \mathrm{~h}$ at $4^{\circ} \mathrm{C}$. After washing with PBS, $50 \mu \mathrm{L}$ of $1 \mathrm{wt} \%$ bovine serum albumin in PBS was added to prevent non-specific binding. All prepared samples were observed using a Zeiss Axio Observer Z1.

\section{Cell culture}

C2C12 myoblast cells were obtained from American Type culture Collection (ATCC) and incubated in Dulbecco's modified Eagle's medium
(DMEM) containing $10 \%$ fetal bovine serum (FBS) and 100 units/mL antibiotic/antimycotic at $37^{\circ} \mathrm{C}$ and $5 \%$ $\mathrm{CO}_{2}$. The cell culture reagents were purchased from Thermo Scientific and Life Technologies.

\section{Immunofluorescence observation of BMP2 conjugated on the cell surface}

For immunofluorescence observation of BMP2 in $\mathrm{C} 2 \mathrm{C} 12$ cells, $1 \times 10^{5}$ cells $/ \mathrm{mL}$ were seeded on the BMP2-immobilized Ppy platform and incubated at 37 ${ }^{\circ} \mathrm{C}$ and in $5 \% \mathrm{CO}_{2}$ overnight. The cells were then easily released from the surface with exposure to an electrical field for $30 \mathrm{~s}$ before being resuspended on a cover slip. After 3 h, FITC-conjugated anti-BMP2 antibody was added to the culture medium and incubated for an additional $4 \mathrm{~h}$. Labeled cells were observed using a Zeiss Axio Observer Z1.

\section{Cell sheet multi-layering}

For cell sheet multi-layering, $\mathrm{C} 2 \mathrm{C} 12$ cells were seeded at a density of $5 \times 10^{4}$ cells on BMP2-immobilized, biotin-doped Ppy (BMP2-Ppy) 
platforms (10 mm width, $10 \mathrm{~mm}$ length) and incubated for five days in culture media to facilitate tight cell-cell connections. Subsequently, Ppy platforms were electrically stimulated with CA at $-0.8 \mathrm{~V}$ for $30 \mathrm{~s}$. Cell sheets were then easily detached from Ppy platforms with gentle shaking and pipette control. The cell sheets were transferred to a new culture dish, and the second and third cell sheets were sequentially suspended on the first cell sheet using the procedures described above.

\section{Cell sheet viability}

The cell sheets recovered from the Ppy platform were suspended on a 12-well culture plate and maintained in complete culture media. To monitor the time-dependent viability of cell sheets, calcein AM and ethidium homodimer- 1 were added to the culture media and incubated for $20 \mathrm{~min}$. The labeled cell sheets were observed using a Zeiss Axio Observer Z1. In addition, recovered cell sheets were also suspended on a 24-well culture plate and incubated in complete culture media. After fabricating 1-layer or 3-layered cell sheets, complete culture media was added to each well and incubated in a $5 \% \mathrm{CO}_{2}$ incubator at $37^{\circ} \mathrm{C}$. After a 24 or $48 \mathrm{~h}$ suspension, the viability of cell sheets was evaluated using the Cell Counting Kit-8 (Dojindo).

\section{Morphological observation of cell sheets}

For morphological observations, $5 \times 10^{4}$ cells were incubated on three types of platform: i) biotin-doped Ppy, ii) biotin-doped Ppy with 100 ng/mL of BMP2 in culture medium, or iii) $100 \mathrm{ng}$ of BMP2-immobilized Ppy. After seven days of incubation, electrical stimulation was applied to the Ppy surface to detach the cell sheets. Hoechst 33341 (Life Technologies) was added to the culture media in which the cell sheets were contained, and this was incubated for $30 \mathrm{~min}$. Labeled cell sheets were observed using a Zeiss Axio Observer Z1.

\section{D Observation of multi-layered cell sheet}

For observation of multi-layered cell sheets, C2C12 cell sheets were recovered by electrical stimulation at $-0.8 \mathrm{~V}$ for $30 \mathrm{~s}$ and placed in the wells of a 12-well culture plate. One cell sheet was then stained with Hoechst $33341(0.5 \mu \mathrm{L} / \mathrm{mL}, 1 \mathrm{~h})$ and another was labeled with Streptavidin-Cy3 (SA-Cy3, $0.5 \mu \mathrm{L} / \mathrm{mL}$, $30 \mathrm{~min}$ ) after cell surface biotinylation by Sulfo-NHS-Biotin $(0.5 \mathrm{mg} / \mathrm{mL}, 15 \mathrm{~min})$. After spreading the Hoechst 33341 stained cell sheet on a cover slip, the SA-Cy3 stained cell sheet was placed onto the first layer of cell sheet. The multi-layered cell sheets were observed in 3D under a Zeiss LSM 710 ConfoCor 3 fluorescence microscope.

\section{Alkaline phosphatase activity (ALP) assay}

ALP activity was measured with an ALP assay kit (BioVision, USA) and according to the manufacturer's instructions. Briefly, $5 \times 10^{4} \mathrm{C} 2 \mathrm{C} 12$ cells were seeded and incubated on three types of platform: i) biotin-doped Ppy, ii) biotin-doped Ppy with 100 $\mathrm{ng} / \mathrm{mL}$ of BMP2 in culture media, or iii) $100 \mathrm{ng}$ of a BMP2-immobilized Ppy platform. After incubation for five days, all groups of cell sheets were recovered by electrical stimulation and fabricated into one-, three-, and five-layered sheets. The cell sheets were then transplanted onto 6-well culture plates and incubated for an additional seven days. Subsequently, cell sheets were lysed using ALP assay buffer for $1 \mathrm{~h}$, and all cell lysates were centrifuged at $13500 \mathrm{rpm}$ and $4{ }^{\circ} \mathrm{C}$ for $10 \mathrm{~min}$. After centrifugation, supernatants were placed in a 96-well plate, $p$-nitrophenyl phosphate was added to each well, and they were incubated for $30 \mathrm{~min}$ at $37^{\circ} \mathrm{C}$. All samples were measured with a microplate reader (Power Wave HT, BioTek) at a wavelength of $405 \mathrm{~nm}$.

\section{Alizarin Red staining}

Alizarin red staining was conducted on fabricated cell sheets according to the standard protocol. C2C12 cells at a density of $5 \times 10^{4}$ were incubated on biotin-doped Ppy or BMP-2 loaded Ppy platforms to produce tight cell-cell junctions. All cells were then recovered from the Ppy surface by electrical stimulation at $-0.8 \mathrm{~V}$ for $30 \mathrm{~s}$ and transplanted into a 6-well culture plate. After $24 \mathrm{~h}$, culture media were exchanged with either DMEM containing 10\% FBS or DMEM containing $2 \mathrm{mM}$ L-glutamine, $50 \mu \mathrm{M}$ ascorbic acid, $20 \mathrm{mM} \beta$-glycerol phosphate, and 10\% FBS. Cell sheets were then incubated for another seven days in a $5 \% \mathrm{CO}_{2}$ incubator at $37^{\circ} \mathrm{C}$. Subsequently, the cell sheets were washed twice with deionized water and stained using $40 \mathrm{mM}$ of an Alizarin red (Sigma-Aldrich, $\mathrm{pH} 4.2$ adjusted with $1 \%$ of ammonium hydroxide) solution for $20 \mathrm{~min}$ at room temperature. Finally, cells were washed with deionized water and observed under a microscope. The calcium deposited area was measured from microscope images using Image J software (NIH, USA).

\section{ACKNOWLEDGMENT}

This research was supported by the Basic Science Research Program through the National Research Foundation of Korea (NRF) funded by the Ministry of Science, ICT, and Future Planning (NRF-2013R1A1A3008096).

\section{COMPETING INTERESTS}

The authors have declared that no competing interest exists. 


\section{REFERENCES}

1. Bortin MM, Gale RP, Kay HE, Rimm AA. Bone marrow transplantation for acute myelogenous leukemia: factors associated with early mortality. J Am Med Assoc. 1983; 249: 1166-75.

2. Lucarelli G, Galimberti M, Polchi P, Angelucci E, Baronciani D, Giardini C, et al. Bone marrow transplantation in patients with thalassemia. N Engl J Med. 1990; 322: 417-21.

3. Speck B, Bortin M, Champlin R, Goldman J, Herzig R, Mcglave P, et al. Allogeneic bone-marrow transplantation for chronic myelogenous leukaemia. Lancet. 1984; 323: 665-8.

4. Tateishi-Yuyama E, Matsubara H, Murohara T, Ikeda U, Shintani S, Masaki H, et al. Therapeutic angiogenesis for patients with limb ischaemia by autologous transplantation of bone-marrow cells: a pilot study and a randomised controlled trial. Lancet. 2002; 360: 427-35.

5. Shimizu T, Sekine H, Isoi Y, Yamato M, Kikuchi A, Okano T. Long-term survival and growth of pulsatile myocardial tissue grafts engineered by the layering of cardiomyocyte sheets. Tissue Eng. 2006; 12: 499-507.

6. Shimizu T, Yamato M, Isoi Y, Akutsu T, Setomaru T, Abe K, et al. Fabrication of pulsatile cardiac tissue grafts using a novel 3-dimensional cell sheet manipulation technique and temperature-responsive cell culture surfaces. Circ Res. 2002; 90: e40-e8.

7. Caicedo-Carvajal CE, Liu O, Remache $Y$, Goy A, Suh KS. Cancer tissue engineering: a novel 3D polystyrene scaffold for in vitro isolation and amplification of lymphoma cancer cells from heterogeneous cell mixtures. J Tissue Eng. 2011: 362326.

8. Seidlits SK, Lee JY, Schmidt CE. Nanostructured scaffolds for neural applications. Future Medicine. 2008; 3: 183.

9. Zhao X, Kim J, Cezar CA, Huebsch N, Lee K, Bouhadir K, et al. Active scaffolds for on-demand drug and cell delivery. Proc Natl Acad Sci. 2011; 108: 67-72.

10. DuRaine GD, Brown WE, Hu JC, Athanasiou KA. Emergence of Scaffold-Free Approaches for Tissue Engineering Musculoskeletal Cartilages. Ann Biomed Eng. 2014: 1-12.

11. Haraguchi Y, Shimizu T, Sasagawa T, Sekine H, Sakaguchi K, Kikuchi T, et al. Fabrication of functional three-dimensional tissues by stacking cell sheets in vitro. Nat Protoc. 2012; 7: 850-8.

12. Matsuda N, Shimizu T, Yamato M, Okano T. Tissue engineering based on cell sheet technology. Adv Mater. 2007; 19: 3089-99.

13. Yamato M, Okano T. Cell sheet engineering. Mater Today. 2004; 7: 42-7.

14. Dvir T, Timko BP, Brigham MD, Naik SR, Karajanagi SS, Levy O, et al. Nanowired three-dimensional cardiac patches. Nat Nanotechnol. 2011; 6: 720-5.

15. Fujita J, Itabashi $Y$, Seki T, Tohyama S, Tamura Y, Sano M, et al. Myocardial cell sheet therapy and cardiac function. Am J Physiol Heart Circ Physiol. 2012; 303: H1169-H82.

16. Shah A, Brugnano J, Sun S, Vase A, Orwin E. The development of a tissue-engineered cornea: biomaterials and culture methods. Pediatr Res. 2008; 63: 535-44.

17. Zani A, Pierro A, Elvassore N, De Coppi P. Tissue engineering: an option for esophageal replacement? Seminars in pediatric surgery; 2009: 57-62

18. Jeon S, Moon JM, Lee ES, Kim YH, Cho Y. An Electroactive Biotin-Doped Polypyrrole Substrate That Immobilizes and Releases EpCAM-Positive Cancer Cells. Angew Chem. 2014; 126: 4685-90.

19. Hong $W Y$, Jeon $S H$, Lee ES, Cho $Y$. An integrated multifunctional platform based on biotin-doped conducting polymer nanowires for cell capture, release, and electrochemical sensing. Biomaterials. 2014; 35: 9573-80.

20. Jeon S, Hong W, Lee ES, Cho Y. High-Purity Isolation and Recovery of Circulating Tumor Cells using Conducting Polymer-deposited Microfluidic Device. Theranostics. 2014; 4: 1123

21. Cabanas-Danés J, Huskens J, Jonkheijm P. Chemical strategies for the presentation and delivery of growth factors. J Mater Chem B. 2014; 2: 2381-94.

22. Crouzier T, Fourel L, Boudou T, Albigès-Rizo C, Picart C. Presentation of BMP-2 from a Soft Biopolymeric Film Unveils its Activity on Cell Adhesion and Migration. Adv Mater. 2011; 23: H111-H8.

23. Otero TF, Martinez JG. Biomimetic intracellular matrix (ICM) materials, properties and functions. Full integration of actuators and sensors. J Mater Chem B. 2013; 1: 26-38. 